\title{
Latissimus dorsi transfer for treatment of irreparable rotator cuff tears
}

\author{
Alexander A. Weening • W. Jaap Willems
}

Received: 2 December 2009 /Revised: 18 January 2010 /Accepted: 19 January 2010 /Published online: 16 February 2010

(C) Springer-Verlag 2010

\begin{abstract}
Massive rotator cuff tendon ruptures are not uncommon in older patients. We propose the transfer of the latissimus dorsi muscle for treatment of irreparable ruptures associated with functional impairment and chronic pain. Five women and 11 men were so treated and reviewed with an average follow-up of 26 months. The mean age was 60 years. Four patients also had subscapularis deficiency. Results were assessed with the Constant score and the Oxford shoulder score. Humeral head position was analysed. Statistical analysis was performed by the Wilcoxon non-parametrical test. The Constant score increased by $24.2 \%(p=0.001)$ with all parameters showing improvement. Nine patients showed improved humeral head positioning in internal rotation. Three of four patients with a deficient subscapularis had unfavourable results. Latissimus dorsi transfer for the treatment of irreparable massive rotator cuff tears leads to a substantial clinical improvement. An intact subscapularis tendon is mandatory.
\end{abstract}

\section{Introduction}

A rupture of one of the shoulder rotator cuff tendons (supraspinatus muscle, subscapularis muscle, teres minor muscle, infraspinatus muscle), also called a cuff lesion, is a frequent problem. Epidemiological studies have shown that rotator-cuff lesions increase with age, with a mean $31 \%$ prevalence of a partial- or full-thickness tear in patients above 50 years of age, a $50 \%$ prevalence above the age of 60 and an increase to an $80 \%$ prevalence above 80 years $[1,2]$.

A. A. Weening $\cdot$ W. J. Willems $(\bowtie)$

Department of Orthopedic Surgery and Traumatology,

Onze Lieve Vrouwe Gasthuis Hospital,

P.O. Box 95500, 1090HM Amsterdam, The Netherlands

e-mail: w.j.willems@olvg.nl
Whereas a supraspinatus tendon rupture does not necessarily cause complaints, massive cuff lesions usually do, with chronic pain and severe disability characterised by loss of strength and function [3].

A massive cuff rupture is a lesion that includes two or more of the rotator cuff tendons [4] and, according to Cofield's definition [5], is greater than $5 \mathrm{~cm}$ in diameter. In most cases, it involves a complete rupture of the supraspinatus and infraspinatus tendons; less frequently the subscapularis and teres minor. An untreated massive cuff lesion leads to pain and loss of function due to cranial migration of the humeral head. The head then articulates with the coraco-acromial arch [6]. These changes can subsequently lead to a progressive deterioration of the joint, the so-called 'cuff rupture arthropathy' [4], osteoarthritis secondary to a massive cuff rupture.

A thorough, timely treatment of the rotator cuff rupture is therefore necessary and both conservative and surgical approaches have been described. Although conservative treatment may yield positive results [7], the long-term outcome has been disappointing [8]. Surgical treatment has become increasingly popular $[9,10]$. Open $[11]$ as well as arthroscopic techniques [12] have shown good results in the treatment of small to large tears. The final recovery of massive cuff ruptures is not as predictable. Massive cuff lesions are often associated with retraction of the tendomuscular unit and degenerative changes of the cuff muscles such as fatty infiltration and atrophy. The chance of recovery of the cuff's integrity is therefore considerably lower [13]. Furthermore, these muscular changes are irreversible [14], leading to an 'irreparable massive cuff lesion'.

When repair of the massive cuff lesion is impossible, there are two treatment modalities which have been published over the past two decades: transposition of the surrounding muscles to replace the rotator cuff function and 


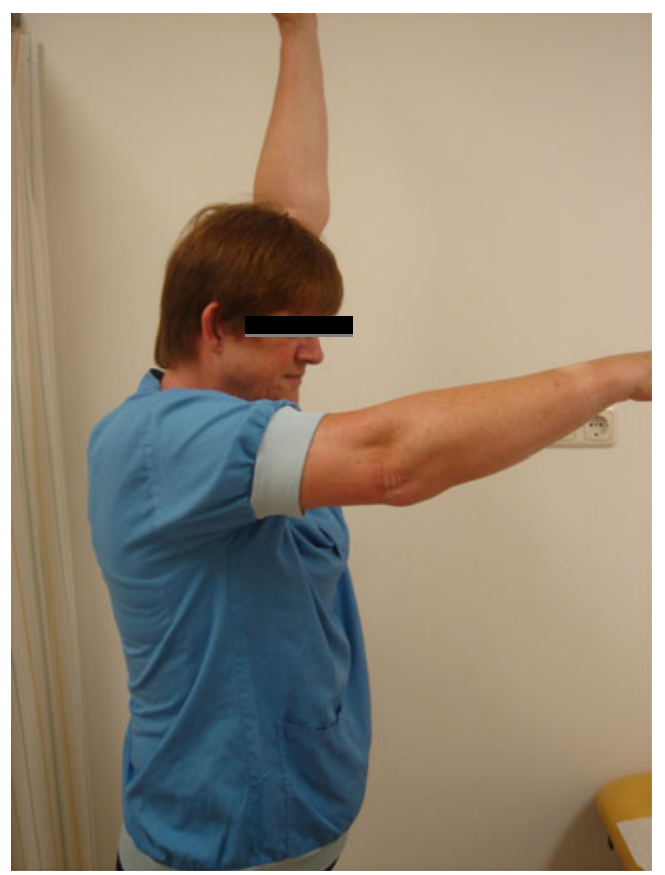

Fig. 1 Patient with a massive cuff lesion shows anteflexion restriction of 90 degrees

a reversed shoulder arthroplasty. In general, a reverse prosthesis is used in a shoulder with so-called 'cuff tear arthropathy', a massive cuff lesion with osteoarthritis. In case of a massive cuff lesion with an intact joint in a symptomatic patient, a tendon transposition is preferred. Tendon transfers, most frequently described in the literature, are those of the deltoid muscle [15], the major pectoral muscle [16], the long head of the triceps [17] and the latissimus dorsi muscle [18-20].

The latissimus dorsi muscle is a large muscle, localised along the dorso-lateral side of the trunk. The muscle has four points of origin: the spinous processes of the thoracic vertebrae (T7-T12), the inferior angle of the scapula, the iliac crest and the ninth to twelfth ribs. By its insertion to the proximal humerus shaft, the muscle contributes to internal rotation, retroversion and abduction of the shoulder joint. L'Episcopo [21] originally described the transposition of the dorsal latissimus muscle in children with a congenital brachial plexus lesion. By moving the latissimus dorsi and the insertion of the teres major to the front of the humeral shaft, the internal rotation contracture could be treated. After transposition of the tendon to the greater tuberosity, the function of the muscle changes into an external rotator and a depressor of the shoulder. Depression of the humeral head, allows the deltoid muscle to contribute to the abduction and anteflexion of the shoulder more effectively.

In this study we present the results of a latissimus dorsi transfer, performed in 16 patients with an irreparable massive cuff lesion.

\section{Material and methods}

Patient data

Of the 20 patients who underwent a latissimus dorsi transposition between 2001 and 2008, 16 were available for follow-up. Four patients were excluded because they could not be reached or decided not to participate in the study.

The remaining group of patients consisted of five women and 11 men. Standard MRI-imaging was performed preoperatively. All patients were diagnosed with at least a rupture of the infraspinatus and supraspinatus tendon, associated with impaired range of motion and pain (Fig. 1). In four patients there was an additional rupture of the subscapularis muscle. In seven patients the ruptures were caused by a trauma, in nine patients no causative factor could be identified. In nine patients a previous open or arthroscopic repair was performed. The mean age at the time of the transposition was 60 years (range 49-71 years).

\section{Surgical procedure}

All patients underwent a transposition of the latissimus dorsi as described by Gerber et al. [18]. The patient was positioned in a lateral decubitus position. Using an Sshaped incision in the axilla area, the insertion of the latissimus dorsi is explored and mobilised (Fig. 2). Subsequently, the tendon is directed between the major teres muscle and the deltoid muscle (Fig. 3) and then, through a separate small mid-deltoid incision, fixed on the footprint of the supraspinatus/infraspinatus area of the greater tuberosity as far anterior as possible. Finally, both wounds are closed in layers and a supporting brace with slight abduction was applied for one week.

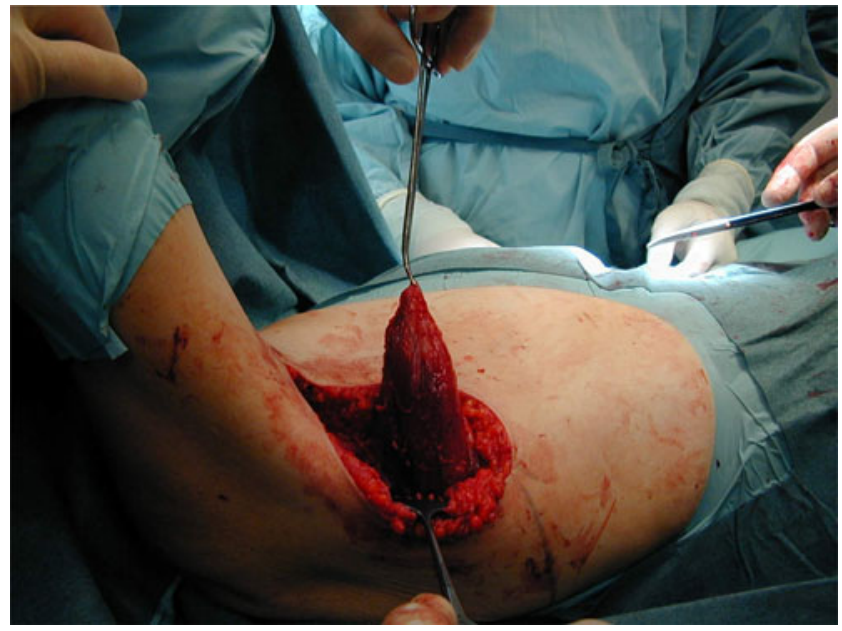

Fig. 2 Intraoperative photo shows the mobilised latissimus dorsi muscle detached from its origin on the proximal humerus shaft. Over the shoulder, another incision is performed, through which a clamp is directed to the distal incision 


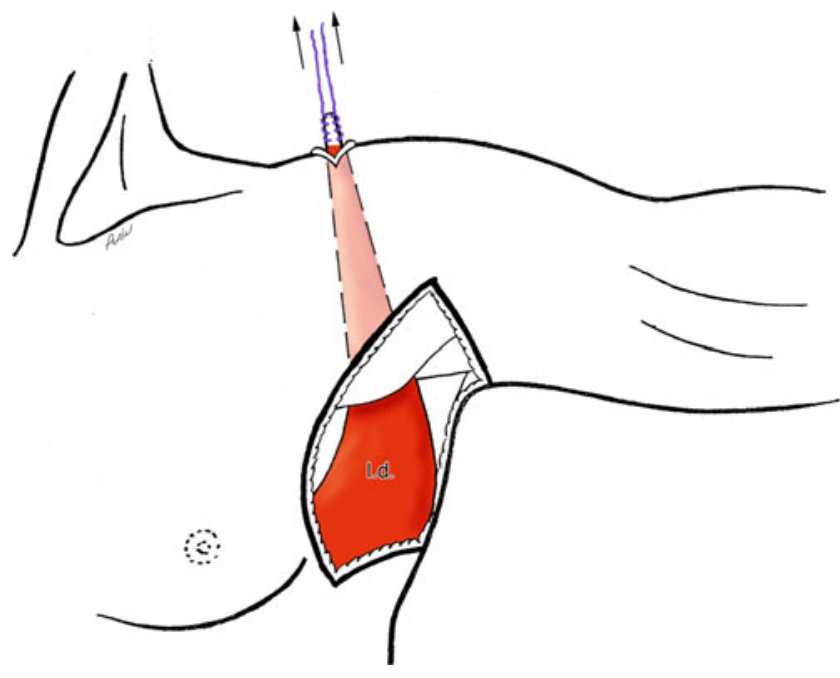

Fig. 3 Between the long head of the triceps muscle and the deltoid muscle, the latissimus dorsi muscle is being led towards the shoulder incision

Postoperative rehabilitation

For a period of six weeks the shoulder was immobilised in a sling after which six weeks of passive physiotherapy was started. From week 12 onwards, active exercises were encouraged.

\section{Evaluation}

All patients were examined clinically in the pre and postoperative phases and were evaluated with the Constant score (CS). Postoperatively, there were also assessed with the Oxford shoulder score, a patient oriented questionnaire to determine the level of pain and function in daily life (ADL).

\section{Radiology}

At the time of follow-up a radiograph of the operated shoulder was made in addition to clinical examination. This radiograph was compared with the preoperative radiograph to evaluate the change of position of the humeral head in relation to the glenoid and the acromion. The change of position was determined by examining the interrupted arch that normally runs from the glenoid rim to the neck of the humeral head.

\section{Statistical analysis}

Statistical analysis of the results was performed by means of the Wilcoxon non-parametric test for paired data. The level of significance of the difference between the pre and postoperative results was set at $p<0.05$.

\section{Results}

All patients were examined postoperatively for a mean follow-up of 26 months (range 7-73 months).

Objective results

The CS showed a mean improvement of $17.8 \%(p=0.001)$, from a preoperative 32.5 points to a postoperative 50.3 points. Corrected for age and sex, the relative CS showed an improvement from $39.1 \%$ to $63.3 \%$, an improvement of $24.2 \%(p=0.001)$. The active anteflexion showed an improvement of 27.0 degrees $(p=0.017)$, and the active abduction an improvement of 31.5 degrees $(p=0.007)$. The active external rotation improved by $26.2 \%(p=0.009)$ and the active internal rotation by $8.1 \%(p=0.127)$. Complaints of pain decreased by $38.0 \%(p=0.008)$ with a mean score from 4.3 points to 10.0 points on a scale of $0-15$.

The level of function in daily life (ADL; sleeping, working and recreation) improved by $27 \%(p=0.007)$. The abduction slightly improved from 3.8 to 5.1 points, an increase of $1 \mathrm{~kg}(p=0.073)$ (Table 1).

\section{Subjective results}

In all but one of the patients, the Oxford shoulder score showed improvement in the domain of pain and function in
Table 1 Pre- and postoperative Constant score results

External and internal rotation presented in points according to the Constant score

\begin{tabular}{llll}
\hline Parameter & Preoperative & Postoperative & $P$-value \\
\hline Pain & $4.3($ range 0-15) & $10($ range 0-15) & 0.008 \\
Function & $78.8^{\circ}\left(\right.$ range $\left.0^{\circ}-150^{\circ}\right)$ & $105.8^{\circ}\left(\right.$ range $\left.0^{\circ}-180^{\circ}\right)$ & 0.017 \\
$\quad$ Anteflexion & $74.3^{\circ}\left(\right.$ range $\left.0^{\circ}-150^{\circ}\right)$ & $105.8^{\circ}\left(\right.$ range $\left.0^{\circ}-180^{\circ}\right)$ & 0.007 \\
$\quad$ Abduction & $3.5($ range 0-8) & $6.1($ range 0-10) & 0.009 \\
$\quad$ External rotation & $5.8($ range $2-10)$ & $6.6($ range $0-10)$ & 0.127 \\
$\quad$ Internal rotation & $3.8($ range 0-13) & $5.1($ range 0-9) & 0.073 \\
Strength & $2.4($ range 0-7) & $5.1($ range 0-10) & 0.007 \\
ADL (activities of daily life) & 39.1 (range 16.0-70) & $63.3($ range 24.0-100) & 0.001 \\
Constant score & & & \\
\hline
\end{tabular}


daily life; only overhead activities such as washing and hanging clothes remained difficult.

\section{Radiology}

Pre and postoperative radiographs of 14 patients were available for analysis of the humerus head position. Only if internally rotated, nine patients showed a postoperatively improved glenohumeral orientation; in five patients the cranial migration of the humerus head was unchanged in every position (Fig. 4).

Two patients showed minimal improvement in the CS and in one patient the score deteriorated. They were revised with implantation of a reversed prosthesis. Two of them had a preoperative rupture of the subscapularis tendon. The remaining two patients with a rupture of the subscapularis tendon had moderate function but considered the clinical situation acceptable because of the lack of pain.

One patient developed a postoperative infection that was treated with drainage and antibiotics. This patient had a moderate CS (48), but refused further treatment. There were no other complications.

\section{Discussion}

A massive cuff lesion is not an uncommon condition affecting the shoulder and is associated with persistent pain and loss of function and strength. When conservative treatment fails, surgical intervention seems to be the most appropriate method of treatment. Because of retraction and irreversible degeneration of the musculo-tenindenous units, re-insertion of the torn tendons is usually not possible. In

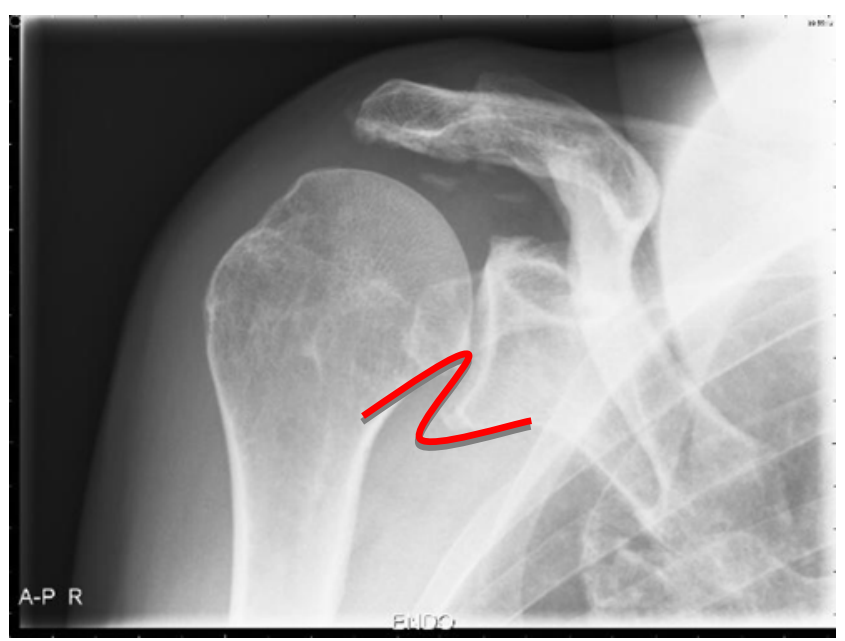

Fig. 4 Radiograph of the right shoulder shows cranial migration of the humeral head compared to the glenoid. An interruption of the arch (glenoid-humeral neck) is identified

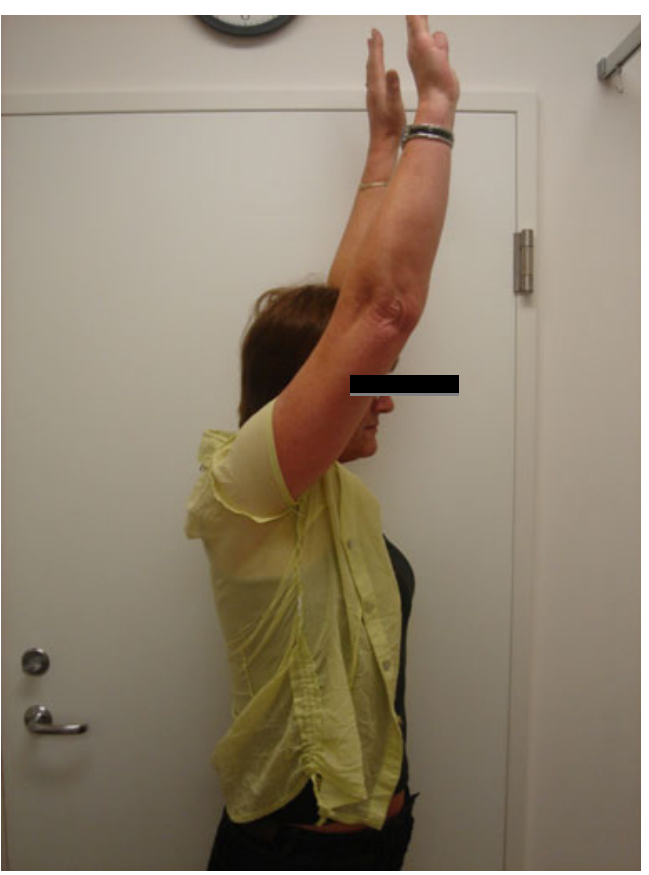

Fig. 5 Latissimus dorsi transposition, one year postoperative; active anteflexion of 160 degrees

these cases transposition of the latissimus dorsi muscle can lead to satisfactory results [22-24]. The transfer initially described by Gerber et al. is the most widely used technique. Single incision techniques have recently been proposed as a sensible alternative with similar results [25].

In our series, transposition by the method of Gerber et al. resulted in an improvement of the mean relative Constant score from $39.1 \%$ to $63.3 \%$, an improvement of $24.2 \%$. This result is similar to the largest study to date, the study of Gerber et al. [22], who showed a Constant score improvement of $18 \%$. All but one, of our patients showed improvement, both in the Constant scores and in the Oxford shoulder scores.

The separate parameters all showed improvement. The largest improvements were obtained in shoulder abduction and anteflexion and in the decrease of pain. Both ranges showed an increase of more than 25 degrees (Fig. 5) and the pain was reduced by $38 \%$. These results were comparable to the data of Gerber et al. [22] who showed a pain decrease of $40 \%$.

The transferred latissimus dorsi is supposed to act as a new effective depressor in restricting the cranial migration of the humeral head. However, postoperative X-rays showed minimal or no depression, especially in the neutral or external rotated position. With internal rotation, nine out of 14 patients showed slightly improved positioning of the humeral head in relation to the glenoid. NoveJosserand et al. [26], expecting a tenodesis effect, discussed a similar hypothesis and had the same position-dependent 
results. It is believed that internal rotation causes an improved tension of the transferred tendon over the humeral head, which could result in a minimal humeral head depression. Five patients showed a persisting cranial migration in every position. Three of them underwent revision. Other studies showed similar results. Gerber et al. [22] did not see a humeral head depression postoperatively. Aoki et al. [23] showed a persisting cranial migration in half of his cases.

With respect to abduction strength, hardly any improvement was noticed. An absolute increase of $1 \mathrm{~kg}(5.5 \%)$, similar to Aoki et al., was seen. Patients noticed that the increase of strength, particularly in overhead activities, remained modest. The inferior gain in strength can be attributed to the inability of the latissimus dorsi to centre the head in the joint as the cuff muscles do, with a resulting inability of the deltoid muscle to regain the pre-existent strength. Patients had been informed about this risk preoperatively. For most patients, this was not considered a problem. An important finding was that daily activities such as shopping or carrying a tray could be performed with decreased or no pain.

Nine patients underwent the transfer as a revision procedure after failed cuff repair. Warner and Parsons found the results to be not as good when the latissimus dorsi transfer was performed in patients who had previous surgery on the rotator cuff and related this to deltoid deficiency [24]. Gerber et al. found nearly comparable results in pain relief and function improvement but also had an inferior outcome after revision [22]. We found little difference in outcome between the revision and primary group (relative CS improvement of $23.88 \%$ vs. $24.47 \%$ ). However, our primary group was relatively small (six patients) and was complicated by different factors (one postoperative infection, two subscapularis tears, two had a history of 'non-cuff repair' shoulder surgery).

Out of four patients with a subscapularis rupture, two underwent revision. One patient with a low score (CS 24) refused re-intervention. Aoki et al., as well as Gerber et al., noted that loss of the subscapularis had a negative effect on the final result due to the prominent role of the subscapularis tendon in positioning of the humeral head in the horizontal and vertical planes. In our study this was confirmed in three out of four patients with a subscapularis rupture. All these patients were operated upon in the early phase of our study, where the relative contraindication for the latissimus dorsi transfer in this group of patients was not yet well known. An intervention that could be considered in cases of simultaneous rupture of the subscapularis is transposition of the pectoralis major muscle.

Exclusion of these four patients resulted in a mean postoperative relative CS of $69.52 \%$.
In conclusion, a latissimus dorsi transposition leads to acceptable results with a low number of complications in patients with irreparable massive cuff lesion. In most patients this procedure leads to a functional improvement of the shoulder with a reduction of pain. The increase of strength is not significant and the patient should be preoperatively informed about this lack of improvement. As has been shown in other studies, transposition usually does not lead to a normal position of the humeral head. An intact subscapular muscle is a prerequisite for this operation.

Acknowledgements We thank Joep Meijer for his expertise and help with the illustrations.

\section{References}

1. Keyes E (1933) Observations on rupture of the supraspinatus tendon: based upon a study of seventy-three cadavers. Ann Surg 97(6):849-856

2. Milgrom C, Schaffler M, Gilbert S, van Holsbeeck M (1995) Rotator-cuff changes in asymptomatic adults. The effect of age, hand dominance and gender. J Bone Joint Surg Br 77(2):296-298

3. Itoi E, Minagawa H, Sato T, Sato K, Tabata S (1997) Isokinetic strength after tears of the supraspinatus tendon. J Bone Joint Surg $\operatorname{Br} 79(1): 77-82$

4. Neer CS 2nd, Craig EV, Fukuda H (1983) Cuff-tear arthropathy. J Bone Joint Surg 65(9):1232-1244

5. Cofield RH (1985) Rotator cuff disease of the shoulder. J Bone Joint Surg 67(6):974-979

6. Visotsky JL, Basamania C, Seebauer L, Rockwood CA, Jensen KL (2004) Cuff tear arthropathy: pathogenesis, classification, and algorithm for treatment. J Bone Joint Surg 86(2):35-40

7. Baydar M, Akalin E, El O, Gulbahar S, Bircan C, Akgul O et al (2009) The efficacy of conservative treatment in patients with fullthickness rotator cuff tears. Rheumatol Int 29(6):623-628

8. Zingg PO, Jost B, Sukthankar A, Buhler M, Pfirrmann CW, Gerber C (2007) Clinical and structural outcomes of nonoperative management of massive rotator cuff tears. J Bone Joint Surg 89 (9):1928-1934

9. Bigliani LU, Cordasco FA, McIlveen SJ, Musso ES (1992) Operative treatment of failed repairs of the rotator cuff. J Bone Joint Surg 74(10):1505-1515

10. O'Holleran JD, Kocher MS, Horan MP, Briggs KK, Hawkins RJ (2005) Determinants of patient satisfaction with outcome after rotator cuff surgery. J Bone Joint Surg 87(1):121-126

11. Zumstein MA, Jost B, Hempel J, Hodler J, Gerber C (2008) The clinical and structural long-term results of open repair of massive tears of the rotator cuff. J Bone Joint Surg 90(11):2423-2431

12. Burkhart SS (2001) Arthroscopic treatment of massive rotator cuff tears. Clin Orthop Relat Res 390:107-118

13. Hersche O, Gerber C (1998) Passive tension in the supraspinatus musculotendinous unit after long-standing rupture of its tendon: a preliminary report. J Shoulder Elbow Surg 7(4):393-396

14. Gladstone JN, Bishop JY, Lo IK, Flatow EL (2007) Fatty infiltration and atrophy of the rotator cuff do not improve after rotator cuff repair and correlate with poor functional outcome. Am J Sports Med 35(5):719-728

15. Lu XW, Verborgt O, Gazielly DF (2008) Long-term outcomes after deltoid muscular flap transfer for irreparable rotator cuff tears. J Shoulder Elbow Surg 17(5):732-737 
16. Elhassan B, Ozbaydar M, Massimini D, Diller D, Higgins L, Warner JJ (2008) Transfer of pectoralis major for the treatment of irreparable tears of subscapularis: does it work? J Bone Joint Surg Br 90(8):1059-1065

17. Malkani AL, Sundine MJ, Tillett ED, Baker DL, Rogers RA, Morton TA (2004) Transfer of the long head of the triceps tendon for irreparable rotator cuff tears. Clin Orthop Relat Res 428:228-236

18. Gerber C, Vinh TS, Hertel R, Hess CW (1988) Latissimus dorsi transfer for the treatment of massive tears of the rotator cuff. A preliminary report. Clin Orthop Relat Res 232:51-61

19. Birmingham PM, Neviaser RJ (2008) Outcome of latissimus dorsi transfer as a salvage procedure for failed rotator cuff repair with loss of elevation. J Shoulder Elbow Surg 17(6):871-874

20. Zafra M, Carpintero P, Carrasco C (2009) Latissimus dorsi transfer for the treatment of massive tears of the rotator cuff. Int Orthop 33(2):457-462

21. L'Episcopo (1939) Restoration of muscle balance in the treatment of obstetrical paralysis. NY State J Med 39:357
22. Gerber C, Maquieira G, Espinosa N (2006) Latissimus dorsi transfer for the treatment of irreparable rotator cuff tears. J Bone Joint Surg 88(1):113-120

23. Aoki M, Okamura K, Fukushima S, Takahashi T, Ogino T (1996) Transfer of latissimus dorsi for irreparable rotator-cuff tears. J Bone Joint Surg Br 78(5):761-766

24. Warner JJ, Parsons IM (2001) Latissimus dorsi tendon transfer: a comparative analysis of primary and salvage reconstruction of massive, irreparable rotator cuff tears. J Shoulder Elbow Surg 10 (6):514-521

25. Lehmann LJ, Mauerman E, Strube T, Laibacher K, Scharf HP (2009) Modified minimally invasive latissimus dorsi transfer in the treatment of massive rotator cuff tears: a two-year follow-up of 26 consecutive patients. Int Orthop. doi:10.1007/s00264-009-0782-5

26. Nove-Josserand L, Costa P, Liotard JP, Safar JF, Walch G, Zilber S (2009) Results of latissimus dorsi tendon transfer for irreparable cuff tears. Orthop Traumatol Surg Res 95(2):108113 